\title{
Hot flashers and He dwarfs in galactic globulars
}

\author{
M. Castellani ${ }^{1}$, V. Castellani ${ }^{1, \star}$, and P. G. Prada Moroni ${ }^{2,3}$ \\ 1 INAF, Osservatorio Astronomico di Roma, via Frascati 33, 00040 Monteporzio Catone, Italy \\ e-mail: m.castellani@oa-roma.inaf.it \\ 2 Dipartimento di Fisica, Università di Pisa, Largo Pontecorvo 3, 56127 Pisa, Italy \\ 3 INFN, Sezione di Pisa, Largo Pontecorvo 3, 56127 Pisa, Italy
}

Received 18 January 2006 / Accepted 1 June 2006

\section{ABSTRACT}

\begin{abstract}
Context. We revisit the evolutionary scenario for hot flasher low-mass structures, where mass loss delays the He flash till the initial phases of their white dwarf cooling sequence.

Aims. Our aim has been to test the theoretical results vis-a-vis different assumptions about the efficiency of mass loss.

Methods. To this purpose, we present evolutionary models covering a fine grid of masses, as obtained assuming a single episode of mass loss in a red giant model of $0.86 M_{\odot}$ with $Z=0.0015$.

Results. We find reasonable agreement with previous evolutionary investigations, showing that for the given metallicity late hot flashers are predicted to cover the mass range $M=0.4975$ to $M=0.4845( \pm 0.0005) M_{\odot}$, all models igniting the He-flash with a mass of the H-rich envelope as given by $M_{\mathrm{e}}=0.00050 \pm 0.00002 M_{\odot}$. The ignition mechanism is discussed in some detail, showing the occurrence of a bifurcation in the evolutionary history of stellar structures at the lower-mass limit for He ignition. Below such a critical mass, the structures miss the He ignition, cooling down as a hot flasher-manqué He white dwarf. We predict that these structures will cool down, reaching the luminosity $\log L / L_{\odot}=-1$ in a time five times longer at the very least than the corresponding cooling time of a normal $\mathrm{CO}$ white dwarf.

Conclusions. On very general grounds, one expects that old stellar clusters with a sizeable population of hot flashers should produce at least a similar amount of slow-cooling He white dwarfs. According to this result, in a cluster where $20 \%$ of red giants escape the He burning phase, one expects roughly twice as white dwarfs than in a normal cluster where all red giants undergo their He flash.
\end{abstract}

Key words. stars: evolution - stars: white dwarfs - stars: mass-loss

\section{Introduction}

Over the past decades the evolution of low-mass stellar structures has been the subject of a large amount of investigations aimed at constraining the evolutionary status of stars in old stellar systems, such as galactic globular clusters. For a long time we have known that the present globular cluster stars are expected to leave their main sequence to climb along the red giant branch (RGB) until the onset of the He-flash. After the phase of central (horizontal branch) and shell (asymptotic giant branch) He-burning phases, they will eventually cool down in the form of carbon-oxygen (CO) white dwarfs (WDs).

In this context, the occurrence of extended "blue tails" in the horizontal branches (HBs) of several galactic globulars has already been understood in terms of RGB structures that have lost a large majority of their H-rich envelope before igniting $\mathrm{He}$ to become HB stars. Castellani \& Castellani (1993; but see also Castellani et al. 1995) found that, for extreme mass loss, there are stellar models that fail to ignite $\mathrm{He}$ at the tip of the RGB, but that undergo a late He-flash during the contraction toward their He-WD structure or in the early stages of the WD cooling sequence. Similar structures are now known in the literature as "hot He-flashers" (HFs). Even higher mass loss will prevent the He ignition, definitively producing He WDs.

Hot flashers have been extensively investigated by several authors. D'Cruz et al. (1996) made use of Reimers (1975, 1977)

* It is with deep sorrow that we announce the death of Vittorio Castellani, who passed away on May 20, 2006. formula for mass loss, taking the efficiency parameter $\eta_{R}$ as a free parameter to explore the range of mass-loss producing HFs for selected assumptions about the star metallicity. Sweigart (1997) discovered that, when the He-flash occurs along the WD cooling sequence ("late" HFs), then convection is expected to reach the H-rich envelope, enhancing $\mathrm{He}$ and Carbon abundances in the stellar atmosphere and driving strong H-flashes. Brown et al. (2001) again adopted the Reimers formalism to explore the occurrence of late HFs for the metal abundance $Z=$ 0.0015 , in connection with observational evidence for extremely hot HB stars in the Galactic globular NGC 2808. Quantitative estimates of the mixing driven by the He-flash have been finally presented by Cassisi et al. (2003), who were able, for the first time, to follow the growth of such an instability in late HFs in detail.

In this paper we revisit the HF theoretical scenario but adopt different assumptions concerning the mass-loss mechanism. On this basis we present and discuss new evolutionary results, focusing attention on the stellar structures marking the transition between late HF and bona fide He-WD.

\section{The models}

All the papers quoted in the previous section have investigated the occurrence of HFs using the Reimers mass-loss parameter $\eta_{R}$ as a free parameter to govern the efficiency and, thus, the amount of mass loss. However, there are subtle differences among these investigations. As a matter of fact Castellani \& Castellani (1993) 
Table 1. Selected physical quantities for stellar structures at the He flash ignition as a function of the mass after the episode of mass loss. Masses and luminosities are in solar units.

\begin{tabular}{lrrrr}
\hline \hline$M$ & $\log L^{\mathrm{f}}$ & $\log T_{\mathrm{e}}^{\mathrm{f}}$ & $M_{\mathrm{c}}^{\mathrm{f}}$ & $M_{\mathrm{e}}^{\mathrm{f}}$ \\
\hline RG Flash & & & & \\
0.5301 & 3.3915 & 3.6221 & 0.49755 & 0.03255 \\
0.5200 & 3.3905 & 3.6287 & 0.49735 & 0.02265 \\
0.5100 & 3.3885 & 3.6388 & 0.49715 & 0.01285 \\
0.5028 & 3.3856 & 3.6580 & 0.49663 & 0.00617 \\
\hline HF-Transition & & & & \\
0.5000 & 3.1639 & 4.8936 & 0.49914 & 0.00086 \\
0.4980 & 3.1745 & 4.9968 & 0.49723 & 0.00077 \\
\hline Late HF & & & & \\
0.4970 & 0.5872 & 4.6049 & 0.49652 & 0.00048 \\
0.4950 & 0.1837 & 4.5160 & 0.49451 & 0.00049 \\
0.4910 & -0.1295 & 4.4438 & 0.49050 & 0.00050 \\
0.4901 & -0.1411 & 4.4388 & 0.48960 & 0.00050 \\
0.4890 & -0.2653 & 4.4122 & 0.48848 & 0.00052 \\
0.4880 & -0.2933 & 4.4038 & 0.48748 & 0.00052 \\
0.4870 & -0.3731 & 4.3914 & 0.48649 & 0.00051 \\
0.4860 & -0.4066 & 4.3907 & 0.48549 & 0.00051 \\
0.4850 & -0.4623 & 4.3917 & 0.48448 & 0.00052 \\
\hline
\end{tabular}

took into account mass loss till the onset of the He-flash, whereas D'Cruz et al. (1996) neglected mass loss when the mass of the H-rich stellar envelope reached the value $M_{\mathrm{e}}=10^{-3} M_{\odot}$. Brown et al. (2001) also stopped the mass loss, but when the structure moved away from the RGB by $\Delta \log T_{\mathrm{e}}[\mathrm{K}]=0.1$.

Here we notice that, at least in principle, these differences can have sizeable consequences on the final structures. Data in Table 4 of Castellani \& Castellani (1993) disclose that, according to Reimers's formulation, after leaving the RGB a HF model is expected to lose an amount of mass within the same order of magnitude as the value of $M_{\mathrm{e}}$ at the onset of the flash $\left(M_{\mathrm{e}}^{\mathrm{f}}\right)$. In turn, theoretical predictions on $M_{\mathrm{e}}^{\mathrm{f}}$ are at the basis of relevant observational constraints, since the minimum value of $M_{\mathrm{e}}^{\mathrm{f}}$ governs the maximum effective temperature that can be reached by normal zero age horizontal branch (ZAHB) models: the maximum effective temperature increases when $M_{\mathrm{e}}^{\mathrm{f}}$ decreases (see, e.g., the discussion in Castellani et al. 1995). It thus obviously appear relevant to investigate theoretical predictions on such a critical issue in detail.

In spite of the different assumptions about mass loss, however, all the investigations we refer to find rather similar values for $M_{\mathrm{e}}^{\mathrm{f}}$. This interesting evidence is supplemented by the result of Brown et al. (2001, but see also D'Cruz et al. 1996), who found that, for a given original chemical composition late HFs all have exactly the same value of $M_{\mathrm{e}}^{\mathrm{f}}$. Such behavior suggests that $M_{\mathrm{e}}$ could be the parameter governing the onset of the flash in HF structures, independent of any assumptions about the mechanism and the efficiency of mass loss. Such a suggestion can be supported by inspection of evolutionary data for HF structures, as given in the already quoted Table 4 in Castellani \& Castellani (1993). Stars leaving the RGB are still supported by CNO H-shell burning. Only when approaching the final ("critical") value of $M_{\mathrm{e}}$ does the CNO burning starts decrease, and the stellar core experiences the final contraction, leading the structure either to a late He-flash or to the final cooling as a He-WD. Here we suggest regarding the quoted time sequence as evidence that in late HFs there is a critical minimum $M_{\mathrm{e}}^{\mathrm{f}}$ value supporting $\mathrm{H}$-shell burning. When this minimum is reached, the H-shell switches-off, causing the core contraction and the switch-on of

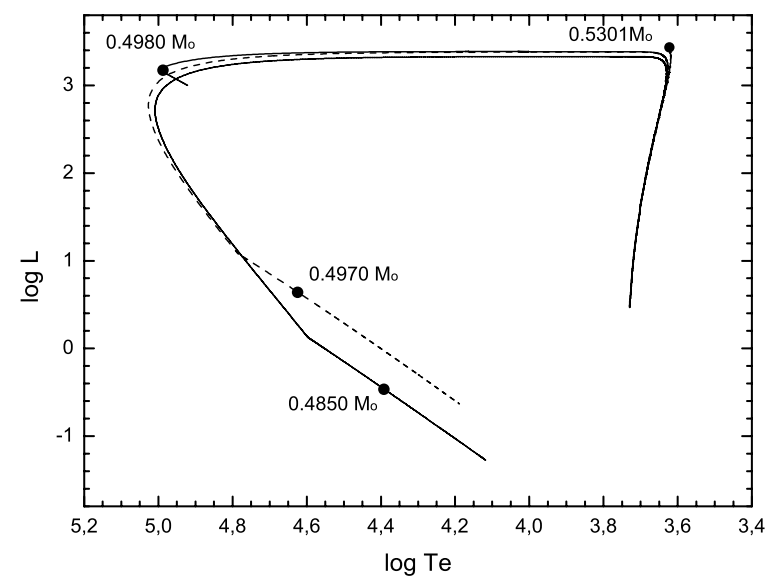

Fig. 1. The evolutionary paths of selected models for the labeled values of the total stellar mass. Dots show the location of the He-flash as given in Table 1 (see text for more details). $L$ is in fraction of $L_{\odot}, T_{\mathrm{e}}$ is in [K].

the He-flash. As is well known, the contrary occurs in normal RGB structures experiencing the He-flash.

To investigate this scenario, we decided to get rid of any interplay between the late evolution of HF models and massloss rates, producing suitable HF progenitors by peeling-off a model during an early stage of RG evolution and thus following the evolution of the structure when assuming no further mass loss. One may notice that a similar procedure has already been adopted by Serenelli et al. (2002). All models were computed assuming an initial helium abundance $Y=0.23$ and a solar-scaled heavy-element abundance $Z=0.0015$, i.e., the same original composition adopted by Brown et al. (2001). If not otherwise stated, in the following we refer to HF progenitors as obtained by applying suitable mass loss to a $0.86 M_{\odot}$ RG structure, when $\log L / L_{\odot}=0.49$ and the mass of the He core $M_{\mathrm{c}}=0.16 M_{\odot}$.

Table 1 gives selected physical quantities for a sample of models experiencing the He-flash. Left to right one finds the mass of the evolving star and, in order, the luminosity, the effective temperature, the mass of the He-core and the mass of the H-rich envelope at the He flash ignition. As already known, one finds that when decreasing the stellar mass the ignition of the He flash moves from the RGB first to the luminous structures crossing the HR diagram toward the WD cooling sequence (transition $\mathrm{HF}$ ) and then to models igniting He along the cooling sequence itself, down to $\log L / L_{\odot} \sim-0.5$ (late HF: LHF). After the He-flash, all the LHF structures have been found to experience the mixing episode and the explosive burning of $\mathrm{H}$ already discussed by Brown et al. (2001) and Cassisi et al. (2003).

The development of the He-flash in HF structures deserves further comment. As is usual, for structures experiencing the Heflash either at the RG tip (RG Flash) or during the crossing of the HR diagram toward the cooling sequence, data in Table 1 refer to the model where the output of the $3 \alpha$ reaction has reached $100 L_{\odot}$ (see, e.g., Sweigart \& Gross 1976). However, for LHFs the identification of the He "flashing" model is less straightforward, since the He ignition has a rather long evolutionary history. Taking the $0.4910 M_{\odot}$ model as an example, the threshold of $L_{3 \alpha}=100 L_{\odot}$ is reached along the cooling sequence at $\log L / L_{\odot}=0.52592$. However, the structure keeps cooling, whereas the efficiency of He burning increases, and the maximum of the $3 \alpha$ production $\left(L_{3 \alpha}=9.2 \times 10^{9} L_{\odot}\right)$ is reached only when $\log L / L_{\odot}=-0.1295$.

According to this evidence, we decided to list data in Table 1 for LHF models at the first maximum of $L_{3 \alpha} / L$. Figure 1 


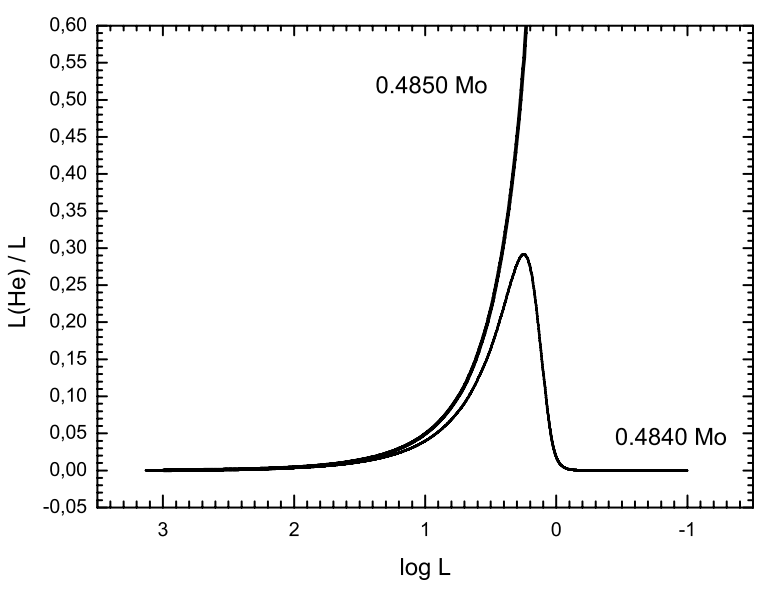

Fig. 2. The He burning contribution to the star luminosity for the two cooling structures at the transition between HF and He white dwarfs (WDs). $L$ is in fraction of $L_{\odot}$.

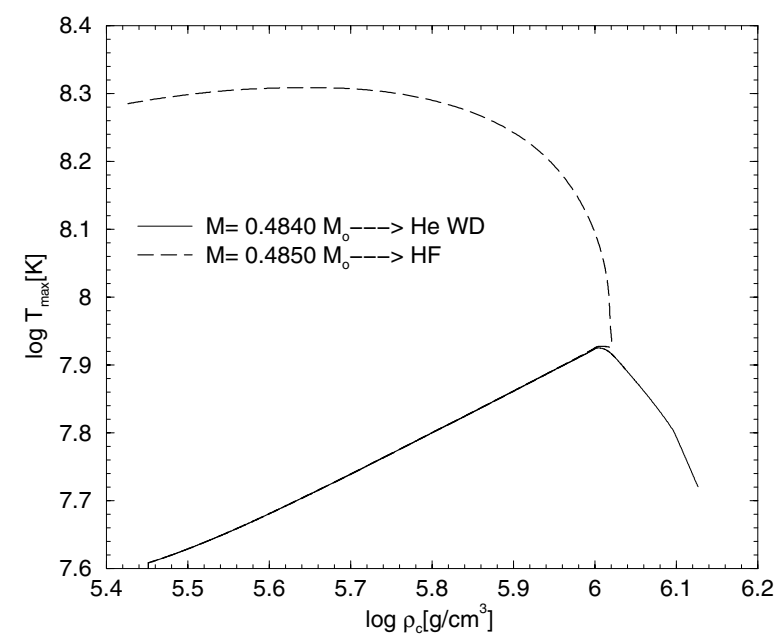

Fig. 3. The run of the maximum (off center) temperature versus the central density for the two cooling structures at the transition between HFs and He WDs.

reports the evolutionary paths of selected HF models. As shown in that figure, the onset of the He-flash in LHF structures is witnessed by a clear discontinuity in the slope of the cooling track. However, the flash attains its maximum after a non negligible evolution along the new cooling curve. As already found by Cassisi et al. (2003), the structure keeps cooling after the flash, until the mixing event causes the $\mathrm{H}$-flashes that drive the star toward its stage of quiescent central He burning.

Inspection of the evolutionary results reveals why the stars miss the He-flash below the critical mass $M=0.485 M_{\odot}$ : decreasing the mass of a HF the contraction driving the efficiency of $3 \alpha$ reactions starts in more advanced phases along the cooling sequence, progressively approaching the region where structures are strongly affected by neutrino emission. Figure 2 shows the contribution of $3 \alpha$ reactions to the star luminosity for the two cooling models of $M=0.4850$ and $0.4840 M_{\odot}$, i.e., just above and below the lower limit for He ignition, at the transition between HF and He WDs. One finds that just below the critical mass the structure starts attempting the final He ignition, but the neutrino emission soon overcomes the output of nuclear energy, cooling down the stellar interior and halting the ignition mechanism. Figure 3 shows the maximum off-center temperature as a function of central density for the same two models. As

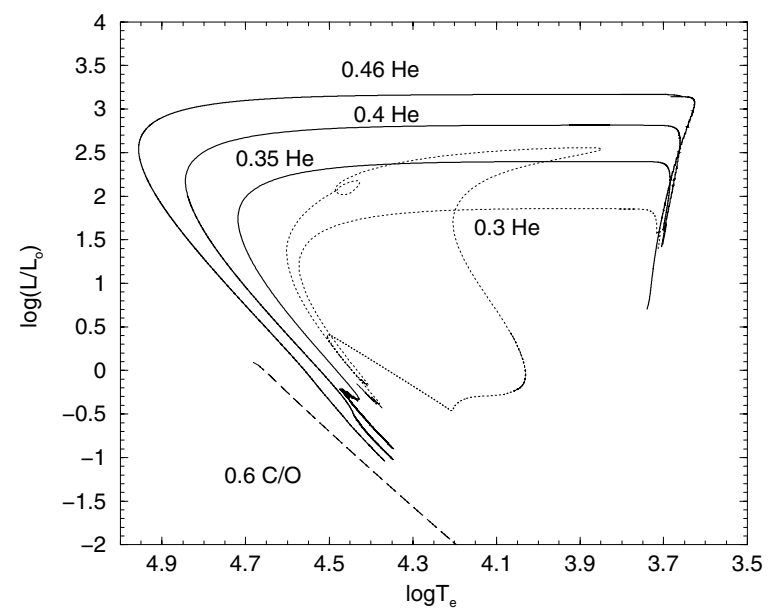

Fig. 4. Evolutionary paths of selected HF-Manqué He WD models for the labelled values of the stellar mass. The cooling sequence of a $0.6 M_{\odot}$ $\mathrm{CO} W D$ is reported for comparison.

already found when discussing the critical stellar mass for carbon ignition (see Fig. 4 in Castellani et al. 2003), even in the case of He ignition one finds clear evidence of a predictable "bifurcation" in the evolution of the physical conditions in the stellar interiors.

Data in Table 1 deserve several comments. One finds that all the LHF reach the flashing phase with a similar envelope mass, namely $M_{\mathrm{e}}=0.00051 \pm 0.00001 M_{\odot}$. This evidence reinforce the suggestion that $M_{\mathrm{e}}$ should be the parameter governing the onset of the late He-flashes. At the same time, this result confirms the evolutionary scenario presented by Brown et al. (2001). Only the mass of the LHF envelopes is slightly smaller in our models, 0.00051 against $0.0006 M_{\odot}$, perhaps for small differences in the input physics (but see also Cassisi et al. 2003 who found $M_{\mathrm{e}}=0.00055 M_{\odot}$ ). Here we notice that the suggested connection between the H-burning switch-off and the onset of late He-flashes gives a natural explanation of the dependence of $M_{\mathrm{e}}^{\mathrm{f}}$ on metallicity (D'Cruz et al. 1996), for which an increase in the metallicity of HF structures causes a decrease in the final pre-flash $M_{\mathrm{e}}$. This indeed appears to agree with the often reported evidence that an increase in the metallicity decreases the minimum mass of the envelope supporting $\mathrm{H}$-shell burning (see, e.g., Castellani et al. 1994).

\section{Hot flashers and He white dwarfs}

According to our computations, one finds a lower-mass limit for the He-flash $M=0.4850 M_{\odot}$ : a decrease in this mass of only $0.001 M_{\odot}$ implies that the stellar structure does not succeed in igniting $\mathrm{He}$, definitely cooling down as a He WD. Figure 4 shows the evolutionary paths for some of these He WD models. As already discussed in Castellani et al. (1994), one finds that, below the critical mass for the onset of the He flash, the more massive WDs are predicted to cool down quietly, at least down to $\log L / L_{\odot}=-1.0$. However, when the WD mass is decreased, the models show the progressive contribution of the H-reignition below $\log L / L_{\odot} \sim 0$, which eventually drives the ignition of strong CNO flashes in the $0.3 M_{\odot}$.

One might notice that the predicted LHFs cover a restricted, but not negligible, range of masses, namely $\Delta M \sim 0.012 M_{\odot}$, against the mass dispersion by $\sim 0.02 M_{\odot}$, often taken as representative of normal HB (Rood 1973). Thus the production of a substantial amount of HF does not require too fine a tuning 
Table 2. Selected physical quantities for stellar structures cooling down as He WDs. Masses and luminosities are in solar units, cooling times $t$ in Myr.

\begin{tabular}{rrrrr}
\hline \hline$M$ & $\log L$ & $t$ & $M_{\mathrm{c}}$ & $M_{\mathrm{e}}$ \\
\hline 0.4840 & 0.0000 & 3.8 & 0.48342 & 0.00058 \\
& -1.0000 & 92.9 & 0.48352 & 0.00048 \\
0.4600 & 0.0000 & 2.1 & 0.45642 & 0.00358 \\
& -1.0000 & 111.4 & 0.45653 & 0.00347 \\
0.4000 & 0.0000 & 0.8 & 0.39834 & 0.00166 \\
& -1.0000 & 174.2 & 0.39859 & 0.00141 \\
\hline
\end{tabular}

of the mass-loss mechanism. This supports the suggestion by Brown et al. (2001) for which LHFs could be at the origin of the hottest group of HBs observed in the globular NGC 2808, as well as of similar structures in $\omega$ Cen (Moehler et al. 2004). As already discussed by D'Cruz et al. (1996), there is no physical bases for predicting the efficiency of mass loss. However, one can hardly believe in a distribution of mass loss producing LHFs but no WDs. In contrast, the occurrence of HFs very likely implies the occurrence of at least a similar amount of stars that miss the He flash, cooling down as He WDs.

According to their larger heat capacity, we know that He WDs evolve more slowly than CO WDs do, the cooling low depending - for each given WD mass - on the mass of the H-rich envelope, as well as on the efficiency of element-diffusion mechanisms. Having established the minimum HF mass within $10^{-3} M_{\odot}$, we simultaneously fixed, within the same uncertainty, the maximum mass allowed for He dwarfs, together with a firm prediction of the mass of the $\mathrm{H}$-rich envelopes in the various cooling structures. It is of interest to investigate the cooling law for these structures in the range of masses just below the HF interval. This appears particularly relevant since current investigations have already detected an unsuspectedly large amount of HFs candidates in clusters like NGC 2808 (Castellani et al. 2006) and $\omega$ Cen (D'Cruz et al. 2002), but also a huge amount of WDs in the latter cluster (Monelli et al. 2005).

Table 2 gives the cooling times for selected HF-manqué He WDs at the two luminosities $\log L / L_{\odot}=0.0$ and -1.0 , together with the corresponding values of the masses of the He-core and of the H-rich envelopes. Evolutionary times are computed starting from the models reaching the maximum effective temperature after leaving the RGB. Inspection of evolutionary times in Table 2 reveals that at the limit $\log L / L_{\odot}=-1$ HF-manqué He WD should have a minimum cooling age on the order of $90 \mathrm{Myr}$, which increases when the mass decreases. Thus, above the quoted luminosity limit HF-manqué He WD have a lifetime within the same order of magnitude as or larger than typical HB stars.

However, one must notice that models in Table 2 do not account for the effect of element diffusion. According to current estimates, the main effect of diffusion in He WDs is to drive the occurrence of strong CNO flashes that can deeply affect the ageluminosity relation (Althaus et al. 2001a,b). Luckily enough, even if diffusion is taken into account, He WD models just below the LHF mass limit seem to escape such an instability, cooling quietly down toward their fainter evolutionary phases (Serenelli et al. 2002). Thus, element diffusion should only have minor effects. This is confirmed by data in Fig. 5, where we compare the age-luminosity relation for two selected models from our sample with a similar model $\left(M=0.449 M_{\odot}\right)$ presented by Serenelli et al. (2002) for $Z=0.001$ and with diffusion taken into account. On the whole, one may conclude that the age given in

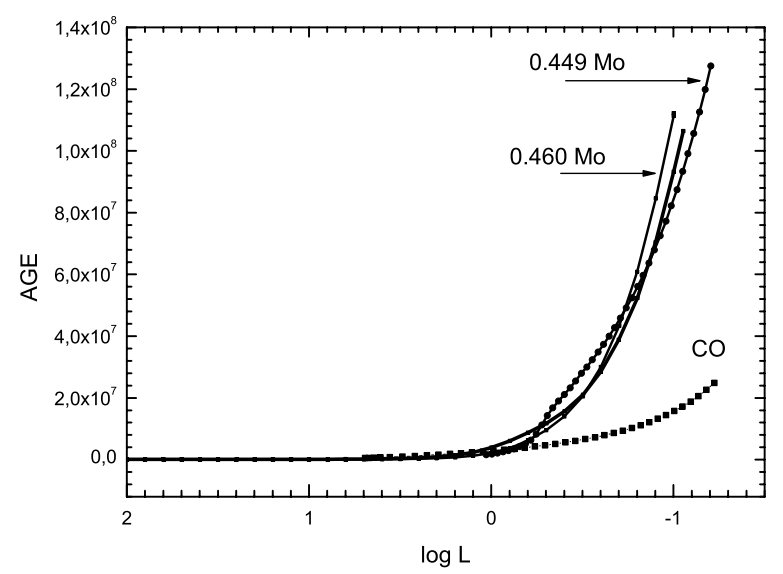

Fig. 5. The cooling ages versus the WD luminosity for our most massive He WD model $\left(0.4840 M_{\odot}\right.$ : heavy line) compared with the less massive model $0.4600 M_{\odot}$, the $0.449 M_{\odot}$ with element diffusion (Serenelli et al. 2002), and a $0.5 M_{\odot}$ CO WD (Prada Moroni \& Straniero 2002). $L$ is a fraction of $L_{\odot}$ and $A G E$ is in years.

Table 2 should give, at least, a reasonable order of magnitude for WD ages.

Comparison with the age-luminosity relation for a CO WD $\left(M=0.5 M_{\odot}\right)$, as given in the same figure from Prada Moroni \& Straniero (2002), offers an impressive evidence of the large predicted differences between HF-manqué He WD and CO WD. One can easily predict that in a cluster where $20 \%$ of the RGs escape the He burning phase, above $\log L / L_{\odot}=-1$ one expects roughly twice as many WD than in a normal cluster where all RGs undergo their He flash. It follows that WD-counts above the quoted luminosity limit can give relevant information on the abundance of HF-manqué He WDs.

\section{Summary and conclusions}

In this paper we have addressed the problem of HF, by investigating the predicted evolutionary behavior of low mass stars with $Z=0.0015$ in detail after an episode of mass loss during their RG evolution. We found that stellar masses in the range $0.485 \leq M \leq 0.497 M_{\odot}$ experience the He flash (and the explosive H-reignition) during their WD cooling phase, when the residual $\mathrm{H}$ shell-burning has reduced the H-rich stellar envelope down to $M_{\mathrm{e}} \sim 0.0005 M_{\odot}$, independently of the mass of the model. Such a result appears to reasonably agree with the theoretical predictions given by Brown et al. (2001) for the same metallicity, the small difference $(M \sim 0.0005$ against $0.0006 M_{\odot}$ ) being the likely the effect of small differences in the adopted input physics, which in turn supports the evidence that the mass of the H-rich envelope plays a critical role in the onset of the delayed He flashes.

According to this result, we also predict the structural parameters needed to evaluate the evolutionary times of structures below the lower-mass limit for He ignition, which will definitely cool down as He WD. We find that these He WD will reach the luminosity $\log L / L_{\odot}=-1$ in a time about 5 times longer than normal CO WDs do, giving a detectable contribution to the abundance of WD above such a luminosity, if and when a not marginal fraction of RG stars escape the He ignition.

For the sake of completeness, one finally has to notice that the current scenario slightly depends on the luminosity of the RG models experiencing the mass loss episode, since peeling $\mathrm{RG}$ structures off either before (as in the previously reported 
computations) or after the first dredge up gives HF progenitors with different $\mathrm{He}$ abundances in the stellar envelopes. Numerical experiments for our $0.86 M_{\odot}$ models have shown that, when the models is stripped after the dredge up $\left(\log L / L_{\odot}=1.48, M_{\mathrm{c}}=\right.$ $0.24 M_{\odot}$ ), the lower-mass limit for LHF moves from 0.485 to $0.491 M_{\odot}$, with LHF models characterized by slightly smaller $\mathrm{H}$-rich envelopes, as given by $M_{\mathrm{e}}^{\mathrm{f}}=0.00048 M_{\odot}$. On the whole, these appear as marginal differences, that do not affect the theoretical scenario we are dealing with.

Acknowledgements. We warmly thank Aldo Serenelli for making models of cooling dwarfs available. It is also a pleasure to thank Santi Cassisi and Giuseppe Bono for a critical reading of the manuscript and for useful comments.

\section{References}

Althaus, L. G., Serenelli, A. M., \& Benvenuto, O. G. 2001a, MNRAS, 323, 471 Althaus, L. G., Serenelli, A. M., \& Benvenuto, O. G. 2001b, MNRAS, 324, 617 Brown, T. M., Sweigart, A. V., Lanz, T., Landsman, W. B., \& Hubeny, I. 2001, ApJ, 562, 368
Cassisi, S., Schlattl, H., Salaris, M., \& Weiss, A. 2003, ApJ, L43

Castellani, M., \& Castellani, V. 1993, ApJ, 407, 649

Castellani, V., Degl'Innocenti, S., \& Pulone, L. 1995, ApJ, 446, 228

Castellani, V., Luridiana, V., \& Romaniello, M. 1994, ApJ, 428, 633

Castellani, V., Degl'Innocenti, S., Marconi, M., Prada Moroni, P. G., \& Sestito, P. 2003, A\&A, 404, 645

Castellani, V., Zoccali, M., Bono, G., et al. 2006, A\&A, in press

D'Cruz, N. L., Dorman, B., Rood, R. T., \& O'Connel, R. W. 1996, ApJ, 466, 359

D'Cruz, N. L., Rood, R. T., O'Connel, R. W., et al. 2002, $\omega$ Centauri, a unique window into astrophysics, ed. F. van Leeuwen, J. D. Huges, \& G. Piotto, ASP Conf. Ser., 265, 235

Moehler, S., Sweigart, A. V., Lndsman, W. B., Hammer, N. J., \& Dreizler, S. 2004, A\&A, 415, 313

Monelli, M., Corsi, C. E., Castellani, V., et al. 2005, ApJ, 621, L117

Prada Moroni, P. G., \& Straniero, O. 2002, ApJ, 581, 585

Reimers, D. 1975, Mem. Royal Soc. Liege, 8, 369

Reimers, D. 1977, A\&A, 57, 395

Rood, R. T. 1973, ApJ, 184, 815

Serenelli, A. M., Althaus, L. G., Rohrmann, R. D., \& Benvenuto, O. G. 2002, MNRAS, 337, 1091

Sweigart, A. V. 1997, Third Conference on Faint Blue Stars, ed. A. G. D. Phillip, J. Liebert, \& R. A. Saffer (Schenrctaday: L.Davis Press), 3

Sweigart, A. V., \& Gross, P. G. 1976, ApJS, 32, 367 